\title{
Geometrical characteristics associated with atherosclerotic disease in the basilar artery: a magnetic resonance vessel wall imaging study
}

\author{
Shasha Deng ${ }^{1 \#}$, Jinmei Zheng ${ }^{1 *}$, Yuxin Wu ${ }^{1}$, Dandan Yang ${ }^{2}$, Huajun Chen $^{1}$, Bin Sun ${ }^{1}$, Yunjing Xue ${ }^{1 *}$, \\ Xihai Zhao ${ }^{2 *}$
}

${ }^{1}$ Department of Radiology, Fujian Medical University Union Hospital, Fuzhou, China; ${ }^{2}$ Department of Biomedical Engineering, School of Medicine, Center for Biomedical Imaging Research, Tsinghua University, Beijing, China

\#These authors contributed equally to this work and are co-first authors.

*These authors contributed equally to this work and are co-corresponding authors.

Correspondence to: Yunjing Xue, MD. Department of Radiology, Fujian Medical University Union Hospital, Fuzhou 350001, China. Email: xueyunjing@126.com; Xihai Zhao, MD, PhD. Center for Biomedical Imaging Research, School of Medicine, Tsinghua University, Haidian District, Beijing 100084, China. Email: xihaizhao@tsinghua.edu.cn.

Background: Vascular geometry may play an important role in the development of atherosclerosis. This study aimed to investigate the relationships between the geometrical characteristics of basilar artery (BA) and the presence, burden, and distribution of BA plaques using magnetic resonance vessel wall imaging.

Methods: Patients with cerebrovascular symptoms in the posterior circulation were recruited and underwent magnetic resonance imaging. The BA's geometrical characteristics, including actual length, straightened length, tortuosity, lateral basilar artery-vertebral artery (VA) angle, lateral mid-BA angle, and BA convexity, were measured. The presence of plaques, stenosis, and plaque burden, including mean and maximal wall thickness, were evaluated. The BA's cross-sectional vessel wall was divided into 4 quadrants: dorsal, ventral, right, and left quadrant. The distribution of BA plaques was analyzed.

Results: Of 344 recruited patients (mean age: 68.1 \pm 11.1 years; 200 males), 100 (29.1\%) had BA plaques. Patients with BA plaques had higher tortuosity of the BA $(13.6 \pm 9.0$ vs. $9.7 \pm 7.7, \mathrm{P}<0.001)$ compared to those without BA plaques. Multivariate regression analysis showed that tortuosity of the BA was associated with the presence of BA plaques (OR, 1.641; 95\% CI, 1.232 to 2.186; $\mathrm{P}=0.001)$ and mean wall thickness $(\beta, 0.045$; $95 \%$ CI, 0.008 to $0.081 ; \mathrm{P}=0.017)$. The plaque distribution in the left wall and right wall of $\mathrm{BA}$ was more frequent in patients with right $(\mathrm{P}=0.006)$ and left $(\mathrm{P}<0.001)$ convex $\mathrm{BA}$, respectively.

Conclusions: The BA's geometrical characteristics, particularly tortuosity and convexity, are independently associated with the presence, burden, and distribution of plaques in the BA.

Keywords: Basilar artery (BA); geometry; atherosclerosis; magnetic resonance imaging

Submitted Nov 21, 2020. Accepted for publication Feb 10, 2021.

doi: $10.21037 /$ qims-20-1291

View this article at: http://dx.doi.org/10.21037/qims-20-1291 


\section{Introduction}

Posterior circulation ischemia accounts for approximately $20-25 \%$ of cerebrovascular ischemic strokes (1). Basilar artery (BA) atherosclerotic plaques are an important cause of posterior circulation ischemic stroke (2). Increasing evidence has shown that the burden and distribution of atherosclerotic plaques are related to cerebrovascular disease risk. Previous studies have reported that plaque burden measured by wall thickening was associated with acute ischemic stroke $(3,4)$. Since BA perforators mainly originate from its lateral or dorsal walls (5), atherosclerotic plaques distributed in the BA lateral wall were more likely to be correlated with symptoms (6). Therefore, it is crucial to better understand the factors associated with the presence, burden, and distribution of atherosclerotic plaques in the BA before occurrence of ischemic events.

Recent studies have utilized vascular geometrical risk factors to explain the presence and characteristics of the burden and distribution of atherosclerotic plaques. For example, high tortuosity was associated with atherosclerotic plaques in the middle cerebral artery (7) and superficial femoral artery (8). High carotid tortuosity was also an independent predictor for plaque burden of the internal carotid artery bulb measured by wall thickness (9). Vascular geometric characteristics may influence local hemodynamic forces and play an important role in the development of atherosclerotic plaques. However, there are geometric variations in the vertebrobasilar system. For example, the angle between the vertebral artery (VA) and BA is different. The left VA is often larger than the right VA (10). Therefore, asymmetric VA flow may induce higher tortuosity in the BA (11). The BA tortuosity and the angle between the VA and BA may influence the hemodynamic patterns associated with the presence and progression of atherosclerotic plaques. Unfortunately, few studies have explored the associations between geometrical characteristics of the BA and the presence, burden (12), and distribution of atherosclerotic plaques in the BA $(13,14)$. The relationship between the BA convexity and the distribution of atherosclerotic plaques is still controversial $(13,14)$. This study's objective was to evaluate the associations between the geometrical characteristics of the BA and the presence, burden, and distribution of atherosclerotic plaques in the BA using high-resolution magnetic resonance (MR) vessel wall imaging.

\section{Methods}

\section{Study population}

In this study, patients who had recent cerebrovascular symptoms (ischemic stroke or transient ischemic attack in the posterior circulation $<2$ weeks) were consecutively enrolled and underwent MR vessel wall imaging for intracranial arteries. The exclusion criteria were as follows: (I) non-atherosclerotic vasculopathy, such as arterial dissection, moyamoya disease and arteritis determined by computed tomography angiography, magnetic resonance angiography (MRA), or MR vessel wall imaging; (II) embolic sources from the heart (atrial fibrillation or valvular heart disease) and the aorta; (III) bilateral VAs did not converge to a common BA congenitally; (IV) occluded BA; (V) contraindication to MR examination. Demographic and clinical information, including age, gender, body mass index (BMI), history of hypertension, diabetes mellitus, hyperlipidemia, smoking status, and coronary heart disease, was collected from medical records. The study protocol was reviewed and approved by the Institutional Review Board, and all subjects provided written informed consent.

\section{MR vessel wall imaging}

MR vessel wall imaging was performed on a 3.0 T MR scanner (Discovery 750, GE Healthcare, Milwaukee, WI, USA) equipped with a 32-channel head coil. The imaging protocol for MR vessel wall imaging included time-of-flight (TOF) MRA and three-dimensional (3D) variable refocusing flip angle fast spin echo T1-weighted imaging (T1-CUBE). The 3D TOF MRA for intracranial arteries was acquired using the following parameters: spoiled gradient recalled, repetition time/echo time $20 / 3.4 \mathrm{~ms}$, the field of view $22 \mathrm{~cm} \times 16.5 \mathrm{~cm}$, flip angle $20^{\circ}$, matrix size $512 \times 192$, slice thickness $1.0 \mathrm{~mm}$, and scan time 4 minutes 33 seconds. The 3D T1-CUBE imaging sequence ( slice $=152$ ) was acquired coronally for the BA and the intracranial segments of VAs using the following parameters: fast spin echo, repetition time/echo time $600 \mathrm{~ms} / 14 \mathrm{~ms}$, the field of view $20 \mathrm{~cm} \times$ $16 \mathrm{~cm}$, matrix size of $320 \times 288$, echo train length 28 , slice thickness $0.8 \mathrm{~mm}$, and scan time 6 minutes 31 seconds.

\section{MR image analysis}

The BA MR vessel wall images were reviewed by two 
trained radiologists with more than 2 years of neurovascular imaging experience with consensus using commercialized software (Vessel Explorer 2, TSimaging Healthcare, Beijing, China). The lumen and wall boundaries of the BA were manually outlined (15). Wall thickness was automatically measured as the average distance between the lumen and wall boundaries. The plaque burden of mean wall thickness (Mean WT) and maximal wall thickness (Maximal WT) was calculated as the average and maximal per artery (16). The atherosclerotic plaque was defined as eccentric or focal wall thickening, while the thinnest part of the wall was estimated to be $<50 \%$ of the thickest point on T1W imaging (17). The degree of BA stenosis was estimated by dividing "blood lumen area" excluding plaques by "vessel inner wall area" and multiplying by 100 (13). The BA crosssectional vessel wall was divided into 4 quadrants: dorsal, ventral, right, and left quadrant (6). For cases with plaques involving more than 2 quadrants, the quadrant with the maximal plaque thickness was chosen. If 1 plaque was equally distributed in 2 quadrants, observers looked at the adjacent proximal and distal slices and determined if there was a majority of quadrant distribution to assist with their assessment.

The methodology for measuring BA geometry was summarized in Figure S1. The geometry of the vertebrobasilar arterial system was evaluated from the following aspects: (I) the actual length; (II) the straightened length; (III) the tortuosity; (IV) the direction of BA convexity; (V) the BA angles from the lateral view (lateral BA-VA angle and lateral mid-BA angle). The actual length and the straightened length of the BA were measured on TOF MRA images. The BA centerline was automatically extracted by tracing the course of the vessel using the MR workstation (Advantage Workstation, AW4.7; GE Medical Systems). The curved segments of the BA were also taken into account. The BA actual length was measured along the centerline from the top of the BA to the junction of both VAs (18). The straightened length was measured by a linear distance between the top of the BA to the junction of both VAs (18). The tortuosity of the BA was measured by the following formula (18): tortuosity = (actual length/ straightened length -1$) \times 100$. A straight line between the top of the BA to the junction of both VAs was used to determine BA convexity (toward the right side or the left side or straight) in the anteroposterior view (13). Two lines were drawn from the vertebrobasilar junction to the BA (a line) and the dominant VA (b line) in the lateral view, and the angle between the 2 lines was regarded as the lateral
BA-VA angle $(13,19)$. The dominant VA was defined as the VA with a larger diameter or connected to the BA in a straighter fashion (11). An imaginary line (c line) was drawn from the posterior cerebral artery bifurcation point, maximally including the $\mathrm{BA}$ in the midline. The point where a line and $c$ line conjoined was regarded as the mid-BA point, and the angle between the two lines was considered as the lateral mid-BA angle (13).

\section{Reproducibility study}

A total of 30 patients were randomly selected for interobserver and intra-observer reproducibility studies. Two observers (DS and WY) independently measured the geometrical characteristics of the BA. One observer (DS) interpreted all the images of the 30 cases again after 1 month time interval, blinded to the results of the first round of review to minimize memory bias.

\section{Statistical analysis}

All statistical analyses were performed using the software SPSS version 22.0 (IBM, Chicago, IL, USA). Continuous variables were summarized as mean \pm SD (standard deviation), and categorical variables were presented as count (percentage). The clinical data and BA geometrical characteristics were compared between subjects with and without BA plaques using the independent $t$ test or Mann-Whitney $U$ test for continuous variables and the Chi-square test for categorical variables. Univariable and multivariable logistic regressions were performed to assess the associations between geometrical characteristics of the BA and the presence of BA plaques. Variables with the potential association $(\mathrm{P}<0.10)$ indicated by the univariable logistic regression were included in the model of multivariate logistic regression. The relationships between the BA geometry and the plaque burden and stenosis of the BA were analyzed using linear regression. The differences in plaque distribution were determined using the MannWhitney $U$ test or Kruskal-Wallis test. The inter-observer and intra-observer reproducibility in measuring BA geometry was evaluated using the intraclass correlation coefficient (ICC). The $\mathrm{P}$ value $<0.05$ was considered as statistically significant.

\section{Results}

A total of 366 patients were enrolled in this study between 
Table 1 Clinical and geometrical characteristics of the study population $(n=344)$

\begin{tabular}{|c|c|c|c|}
\hline Characteristics & \multicolumn{2}{|c|}{ Mean \pm SD or $n(\%)$} & $P$ value \\
\hline \multicolumn{4}{|l|}{ Clinical characteristics } \\
\hline Age, years old & $70.5 \pm 11.3$ & $67.1 \pm 10.8$ & 0.006 \\
\hline Gender, male & $60(60.0)$ & $140(57.4)$ & 0.654 \\
\hline Hypertension & $84(84.0)$ & $157(64.3)$ & $<0.001$ \\
\hline Diabetes & $49(49.0)$ & 73 (29.9) & 0.001 \\
\hline Hyperlipidemia & $30(30.0)$ & $68(27.9)$ & 0.691 \\
\hline Smoker & $35(35.0)$ & $85(34.8)$ & 0.977 \\
\hline Actual length (cm) & $2.8 \pm 0.5$ & $2.7 \pm 0.4$ & 0.258 \\
\hline Straightened length (cm) & $2.5 \pm 0.4$ & $2.5 \pm 0.3$ & 0.446 \\
\hline Tortuosity & $13.6 \pm 9.0$ & $9.7 \pm 7.7$ & $<0.001$ \\
\hline Lateral BA-VA $\left({ }^{\circ}\right)$ & $24.0 \pm 21.9$ & $24.7 \pm 22.7$ & 0.828 \\
\hline Lateral mid-BA $\left(^{\circ}\right)$ & $28.7 \pm 15.5$ & $28.4 \pm 13.4$ & 0.832 \\
\hline
\end{tabular}

$\mathrm{SD}$, standard deviation; BA, basilar artery; BMI, body mass index; VA, vertebral artery.

May 2017 and August 2020 from our hospital. Of the 366 patients, 22 were excluded due to the following reasons: (I) poor image quality ( $\mathrm{n}=7$ ); (II) bilateral VAs did not converge to the BA congenitally ( $\mathrm{n}=9$ ); and (III) BA with total occlusion $(\mathrm{n}=6)$. Of the remaining 344 patients (mean age: $68.1 \pm 11.1$ years old; 200 males), $100(29.1 \%)$ had atherosclerotic plaques in the BA, of which $10(10.0 \%)$ patients had concentric plaques, $90(90 \%)$ patients had eccentric plaques, and 13 patients had calcified plaques in BA. Compared to patients without BA plaques, those with BA plaques had older age $(70.5 \pm 11.3$ vs. $67.1 \pm 10.8$ years, $\mathrm{P}=0.006)$, a higher incidence of hypertension $(84.0 \%$ vs. $64.3 \%, \mathrm{P}<0.001)$ and diabetes $(49 \%$ vs. $29.9 \%, \mathrm{P}=0.001)$, and greater tortuosity of the BA $(13.6 \pm 9.0$ vs. $9.7 \pm 7.7$, $\mathrm{P}<0.001)($ Table 1).

\section{Associations between geometry and the presence of plaques in the $B A$}

Univariable logistic regression analysis showed that age (OR, 1.029; 95\% CI, 1.006 to 1.052 ; $\mathrm{P}=0.012$ ), hypertension
(OR, 2.909; 95\% CI, 1.604 to 5.276; $\mathrm{P}<0.001$ ), diabetes (OR, 2.251; 95\% CI, 1.395 to $3.631 ; \mathrm{P}=0.001$ ), and the tortuosity of the BA (OR, 1.592; 95\% CI, 1.250 to 2.028; $\mathrm{P}<0.001)$ were significantly associated with the presence of plaques. After adjusting for confounding factors including age, hypertension, diabetes, and the actual length of the $\mathrm{BA}$, the association between BA plaques and the tortuosity of the BA (OR, 1.641; 95\% CI, 1.232 to $2.186 ; \mathrm{P}=0.001)$ remained statistically significant (Table 2).

\section{Associations between geometry and plaque burden and stenosis in the $B A$}

Linear regression analysis revealed that the tortuosity of the BA was significantly correlated with Mean WT $(\beta, 0.045$; $95 \%$ CI, 0.008 to $0.081 ; \mathrm{P}=0.017$ ) after adjusting for the confounding factors of age, gender, BMI, smoking status, and hypertension. However, there were no significant correlations between other geometrical characteristics and the plaque burden and stenosis of the BA (all $\mathrm{P}>0.05$, Table 3). 
Table 2 Associations between geometrical characteristics and the presence of plaques in the BA

\begin{tabular}{|c|c|c|c|c|}
\hline Characteristics & \multicolumn{2}{|c|}{ Univariate logistic regression } & \multicolumn{2}{|c|}{ Multivariate logistic regression } \\
\hline \multicolumn{5}{|l|}{ Clinical characteristics } \\
\hline Age, years old & $1.029(1.006,1.052)$ & 0.012 & $1.019(0.995,1.043)$ & 0.121 \\
\hline Gender, male & $1.114(0.694,1.790)$ & 0.654 & & \\
\hline Hypertension & $2.909(1.604,5.276)$ & $<0.001$ & $2.499(1.345,4.644)$ & 0.004 \\
\hline Diabetes & $2.251(1.395,3.631)$ & 0.001 & $1.981(1.195,3.284)$ & 0.008 \\
\hline Hyperlipidemia & $1.109(0.665,1.849)$ & 0.691 & & \\
\hline Smoker & $1.007(0.618,1.641)$ & 0.977 & & \\
\hline Actual length (cm) & $1.576(0.931,2.666)$ & 0.090 & $0.852(0.439,1.656)$ & 0.637 \\
\hline Straightened length $(\mathrm{cm})$ & $0.954(0.471,1.932)$ & 0.895 & & \\
\hline Tortuosity & $1.592(1.250,2.028)$ & $<0.001$ & $1.641(1.232,2.186)$ & 0.001 \\
\hline Lateral BA-VA $\left(^{\circ}\right)$ & $0.999(0.988,1.009)$ & 0.803 & & \\
\hline Lateral mid-BA $\left(^{\circ}\right)$ & $1.002(0.985,1.019)$ & 0.832 & & \\
\hline
\end{tabular}

BA, basilar artery; OR, odds ratio; BMI, body mass index; VA, vertebral artery.

Table 3 Associations between geometrical characteristics and plaque burden and stenosis in the BA

\begin{tabular}{|c|c|c|c|c|c|c|}
\hline & \multicolumn{2}{|l|}{ Mean WT } & \multicolumn{2}{|l|}{ Maximal WT } & \multicolumn{2}{|l|}{ Stenosis } \\
\hline Tortuosity & $0.045(0.008,0.081)$ & 0.017 & $0.007(-0.001,0.015)$ & 0.106 & $-0.080(-0.660,0.499)$ & 0.784 \\
\hline Lateral BA-VA $\left(^{\circ}\right)$ & $0.001(-0.001,0.002)$ & 0.416 & $0.000(-0.003,0.004)$ & 0.937 & $0.052(-0.160,0.264)$ & 0.628 \\
\hline Lateral mid-BA $\left({ }^{\circ}\right)$ & $0.006(-0.032,0.043)$ & 0.768 & $-0.000(-0.005,0.005)$ & 0.992 & $0.250(-0.075,0.575)$ & 0.130 \\
\hline
\end{tabular}

Mean WT, mean wall thickness; Maximal WT, maximal wall thickness; BA, basilar artery; VA, vertebral artery.

\section{Associations between geometry and the distribution of plaques in the $B A$}

From the anteroposterior view, the plaque distribution in the left wall of BA was more frequent in patients with right convex BA than those with a left convex or straight BA (46.2\% vs. $5.9 \%$ vs. $25.8 \%, \mathrm{P}=0.006$ ). In contrast, BA plaque distribution in the right wall was more frequent in patients with left convex BA than in those with a right convex and straight $\mathrm{BA}(58.8 \%$ vs. $9.6 \%$ vs. $25.8 \%, \mathrm{P}<0.001)$ (Table 4 and Figure 1).

From the lateral view, the mid-BA angle was marginally higher in patients with plaques in the dorsal wall than in patients with plaques in the ventral wall $\left(33.4^{\circ} \pm 20.2^{\circ}\right.$ vs. $\left.25.6^{\circ} \pm 12.3^{\circ}, \mathrm{P}=0.086\right)$. There were no significant correlations between the lateral BA-VA angle and the distribution of BA plaques in the ventral and dorsal wall $\left(21.6^{\circ} \pm 19.9^{\circ}\right.$ vs. $\left.23.8^{\circ} \pm 22.9^{\circ}, \mathrm{P}=0.802\right)$.

\section{Reproducibility}

Excellent inter-observer (ICC: $0.875-0.965$ ) and intraobserver (ICC: $0.865-0.935$ ) agreements were found in 
Table 4 Associations between BA convexity and the distribution of plaques in the BA

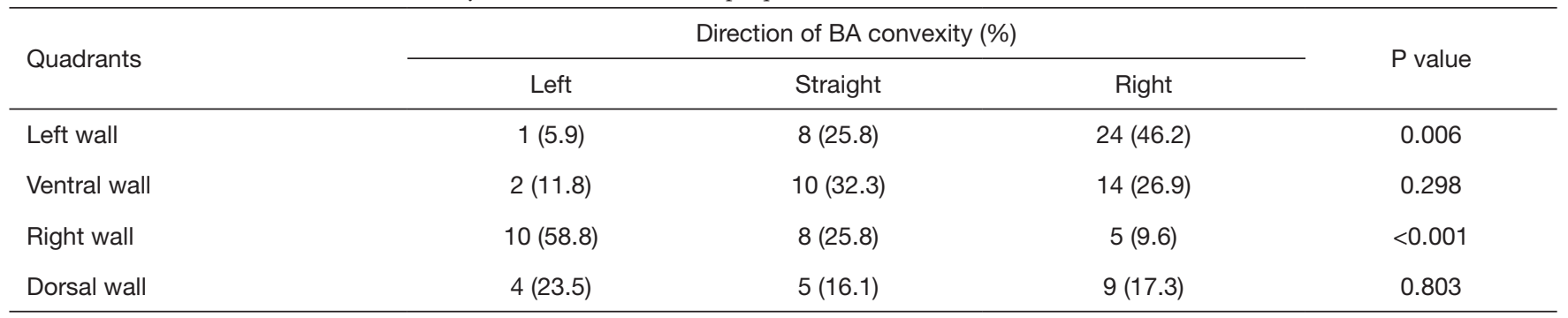

BA, basilar artery.
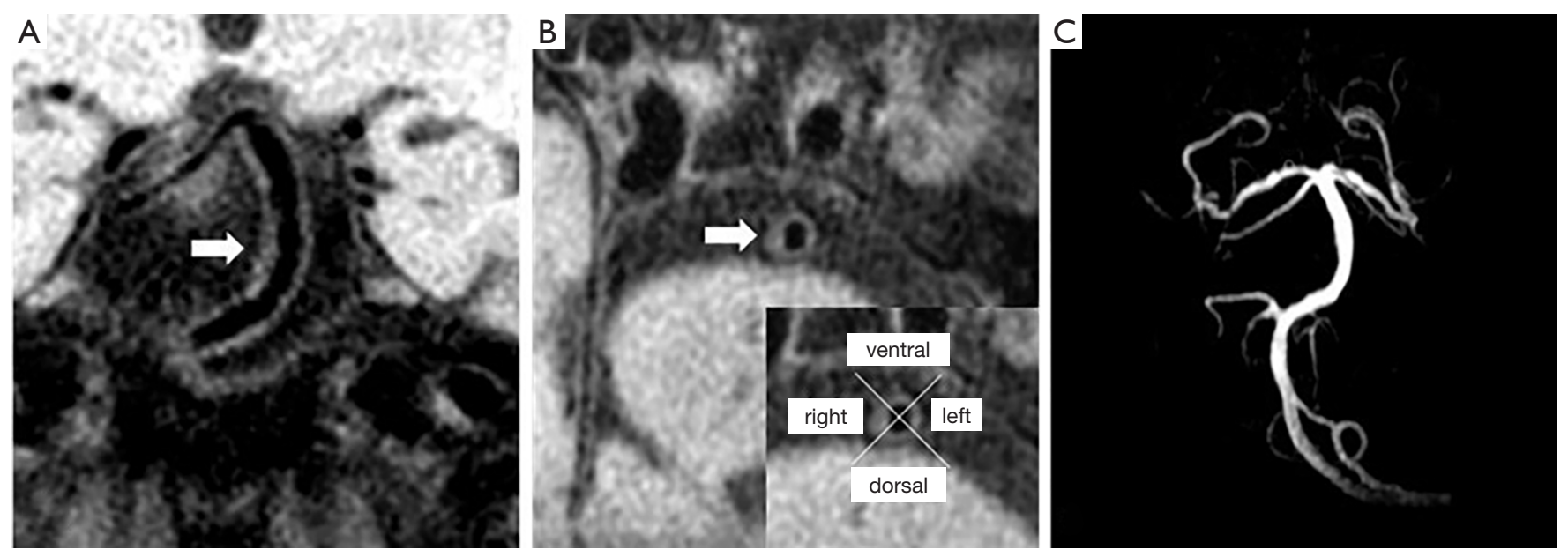

Figure 1 The patient has an atherosclerotic plaque in the basilar artery. The multiple planar reconstructed vessel wall image (A) shows an atherosclerotic plaque (arrow) in the right wall of the BA. Cross-sectional image (B) confirmed that the atherosclerotic plaque (arrow) was located in the right wall. This patient had left convex BA and larger tortuosity (C). BA, basilar artery.

measuring actual length, straightened length, tortuosity, lateral BA-VA angle, and mid-BA angle (Table S1).

\section{Discussion}

This study investigated the relationships between the BA geometrical characteristics and the presence, burden, and distribution of atherosclerotic plaques in the BA using MR vessel wall imaging. We found that the BA tortuosity was associated with the presence and burden (Mean WT) of BA plaques. Moreover, the convexity of the BA was found to be associated with the distribution of plaques. The presence of left plaques was more frequent in patients with right convex BA, whereas the presence of right plaques was more frequent in patients with left convex BA. Our results provide further compelling evidence that geometrical characteristics may play a role in atherosclerosis development and progression.

This study demonstrated that the tortuosity of the BA was associated with the presence of BA plaques. Our results were consistent with previous studies, in which tortuosity was related to middle cerebral artery atherosclerosis (OR, $1.060 ; \mathrm{P}=0.003)(7)$ and the fraction of high curvature of the superficial femoral artery was significantly larger in patients with atherosclerosis than in atherosclerosis-free patients $(22.77 \pm 10.22$ vs. $11.39 \pm 6.82 ; \mathrm{P}<0.001)$ (8). The association between tortuosity and the presence of plaques may be explained by low wall shear stress (WSS). As an important hemodynamic parameter, the WSS represents a frictional force that is parallel to the vessel wall by the viscosity of blood (20). Investigators believed that curvatures of vessels were susceptible to low WSS (21) and higher curvature of vessels had overall larger low WSS compared with lower curvature of vessels $(4.13 \pm 1.91$ vs. $1.79 \pm 1.13, \mathrm{P}=0.009)(8)$. 
Low WSS has been proven to negatively affect endothelial function (22) and stimulate the expression of an atherogenic phenotype (23). However, the WSS of the BA was not studied in the present work. Our results suggest that tortuosity of the BA may play a role in the development of BA atherosclerosis.

We also found that the tortuosity of the BA was significantly correlated with the Mean WT of the BA. Mean WT represents one of the metrics for plaque burden $(24,25)$. Previous studies showed that higher curvature of the superficial femoral artery might lead to larger area ratios of low WSS (8). Low WSS was associated with a greater increase in plaque burden due to both changes in plaque area and the differential pattern of arterial remodeling in the coronary artery (26). Also, a study of mouse carotid arteries showed that the areas of atherosclerotic lesions in the lowered shear stress regions were much larger than those in the regions with vortices and oscillatory shear stress (intima/media, $1.38 \pm 0.68$ vs. $0.22 \pm 0.04 ; \mathrm{P}<0.05)(27)$. Therefore, the higher tortuosity we observed in the BA with larger plaque burden probably indicates lower WSS. However, the relationship between WSS and plaque burden is still controversial $(28,29)$, and the underlying mechanism remains unclear. The potential relationship between higher tortuosity and WT has also been observed in a previous study (9), in which high carotid tortuosity was an independent predictor for the WT of the internal carotid artery bulb (regression coefficient $=0.764 ; \mathrm{P}<0.001$ ). Unlike Zhou et al.'s study (12), our results also showed a significant correlation between BA tortuosity and plaque burden. This discrepancy in the results may be due to the differences in the methodology for measuring vascular tortuosity and the sample size (Zhou et al.'s study: $\mathrm{N}=67$ ). In Zhou et al.'s study, investigators measured vascular tortuosity on the BA maximum angle by subjective inspection visually on MRA. In contrast, our vascular tortuosity was automatically calculated, which reduced subjective bias from the observers. Therefore, given the previous inconsistent observations and the highly artery-specific anatomical structure characteristics, the association between tortuosity and plaque burden requires further determination.

We found that BA plaques were frequently distributed in the lateral walls $(56.0 \%)$ in symptomatic patients. Our results were consistent with Chen et al.'s reports (6), in which BA plaques were frequently located in the lateral walls $(66.1 \%)$. Our findings that patients with right convex BA were more likely to develop atherosclerotic plaques in the left wall and vice versa may provide insights into the underlying mechanisms of BA lateral wall susceptibility to atherosclerotic disease. In the present study, the BA convexity was found to be associated with the distribution of plaques, and the BA plaques were predominantly located in the vessel wall of the inner curvature. A previous study showed that plaques were more often observed at the vessel's inner curvature, probably due to the relatively lower WSS at these locations (30). Our results were consistent with Kim et al.'s study, in which right and left plaques of the BA were more frequently observed in the left $(\mathrm{P}=0.009)$ and right $(\mathrm{P}=0.024)$ convex $\mathrm{BA}$ in an asymptomatic $\mathrm{BA}$ plaque population, respectively (13). The main differences between Kim et al.'s study and our study are the study populations and the study focus. Kim et al.'s study's major focus was the associations between plaque quadrant distribution and BA morphology and the characteristics of plaque burden in an asymptomatic BA plaque population. Beyond evaluating the distribution of BA plaques, our study also investigated the relationship between the BA's tortuosity and plaque formation and plaque burden in a symptomatic BA plaque population. A previous study reported that the location of the pontine infarct was opposite to the convexity of the BA $(\mathrm{P}<0.01)(11)$. These findings suggest that assessment of vascular morphology may be useful for the early prediction of the presence of BA plaques and subsequent ischemic events in the posterior circulation territory.

In addition, we found that hypertension and diabetes were significantly associated with the presence of BA plaques in the present study. Our results were in line with previous studies, in which hypertension (OR, 1.826; $95 \%$ CI, 1.274 to $2.618 ; \mathrm{P}=0.001)$ and diabetes mellitus (OR, 1.490; 95\% CI, 1.105 to $2.010 ; \mathrm{P}=0.009)$ were related to posterior circulation atherosclerosis (31). It has been demonstrated that elevated blood pressure may stimulate the development of atherosclerosis, as elevated blood pressure can damage the endothelium and arterial wall through both mechanical and humoral factors (32). Some investigators revealed that blood glucose disorder was related to oxidative stress, inflammation, endothelial dysfunction, and vasorum neovascularization $(33,34)$.

Several limitations in our study need to be acknowledged. First, the potential influence of existing atherosclerosis on measurements of geometric parameters cannot be completely avoided. The causal relationships between BA geometry characteristics and the presence and burden of plaques need to be demonstrated in a longitudinal study. Second, there was no direct study of intravascular hemodynamics in our work. In the future, non-invasive 
quantitative research on intravascular hemodynamics is needed to bridge the correlations between the geometrical characteristics of the $\mathrm{BA}$ and plaque formation and progression. Third, only five BA geometric parameters were included in this study. Therefore, future work that includes other geometric parameters (vertebrobasilar junction) is warranted. Fourth, because the sample size of calcified plaques was relatively small, we will explore the relationships between geometrical characteristics and plaque composition (calcification) in future work.

\section{Conclusions}

Geometrical characteristics of the BA, particularly tortuosity and convexity, are independently associated with the presence, burden, and distribution of atherosclerotic plaques in the BA. Longitudinal studies are warranted for validation.

\section{Acknowledgments}

Funding: This research was supported by National Key R\&D Program of China (Grant number: 2017YFC1307900, 2017YFC1307904), Joint Funds for the innovation of science and Technology, Fujian province (Grant number: 2018Y9025), and National Natural Science Foundation of China (Grant number: 81771825).

\section{Footnote}

Conflicts of Interest: All authors have completed the ICMJE uniform disclosure form (available at http://dx.doi. org/10.21037/qims-20-1291). The authors have no conflicts of interest to declare.

Ethical Statement: This study was approved by the Ethics Committee of Union Medical College Hospital Affiliated to Fujian Medical University and informed consent was obtained from all the patients.

Open Access Statement: This is an Open Access article distributed in accordance with the Creative Commons Attribution-NonCommercial-NoDerivs 4.0 International License (CC BY-NC-ND 4.0), which permits the noncommercial replication and distribution of the article with the strict proviso that no changes or edits are made and the original work is properly cited (including links to both the formal publication through the relevant DOI and the license). See: https://creativecommons.org/licenses/by-nc-nd/4.0/.

\section{References}

1. Sparaco M, Ciolli L, Zini A. Posterior circulation ischemic stroke-a review part II: imaging and acute treatment. Neurol Sci 2019;40:2007-15.

2. Bogousslavsky J, Regli F, Maeder P, Meuli R, Nader J. The etiology of posterior circulation infarcts: a prospective study using magnetic resonance imaging and magnetic resonance angiography. Neurology 1993;43:1528-33.

3. Lee WJ, Choi HS, Jang J, Sung J, Kim TW, Koo J, Shin YS, Jung SL, Ahn KJ, Kim BS. Non-stenotic intracranial arteries have atherosclerotic changes in acute ischemic stroke patients: a 3T MRI study. Neuroradiology 2015;57:1007-13.

4. Ryu CW, Jahng GH, Kim EJ, Choi WS, Yang DM. High resolution wall and lumen MRI of the middle cerebral arteries at 3 tesla. Cerebrovasc Dis 2009;27:433-42.

5. Marinković SV, Gibo H. The surgical anatomy of the perforating branches of the basilar artery. Neurosurgery 1993;33:80-7.

6. Chen Z, Liu AF, Chen H, Yuan C, He L, Zhu Y, Guan $M$, Jiang WJ, Zhao X. Evaluation of basilar artery atherosclerotic plaque distribution by $3 \mathrm{D}$ MR vessel wall imaging. J Magn Reson Imaging 2016;44:1592-9.

7. Kim BJ, Kim SM, Kang DW, Kwon SU, Suh DC, Kim JS. Vascular tortuosity may be related to intracranial artery atherosclerosis. Int J Stroke 2015;10:1081-6.

8. Li X, Liu X, Li X, Xu L, Chen X, Liang F. Tortuosity of the superficial femoral artery and its influence on blood flow patterns and risk of atherosclerosis. Biomech Model Mechanobiol 2019;18:883-96.

9. Strecker C, Krafft AJ, Kaufhold L, Hüllebrandt M, Weber S, Ludwig U, Wolkewitz M, Hennemuth A, Hennig J, Harloff A. Carotid geometry is an independent predictor of wall thickness - a 3D cardiovascular magnetic resonance study in patients with high cardiovascular risk. J Cardiovasc Magn Reson 2020;22:67.

10. Kazui S, Kuriyama Y, Naritomi H, Sawada T, Ogawa M, Maruyama M. Estimation of vertebral arterial asymmetry by computed tomography. Neuroradiology 1989;31:237-9.

11. Hong JM, Chung CS, Bang OY, Yong SW, Joo IS, Huh K. Vertebral artery dominance contributes to basilar artery curvature and peri-vertebrobasilar junctional infarcts. J Neurol Neurosurg Psychiatry 2009;80:1087-92. 
12. Zhou L, Yan Y, Du H, Ni X, Wang G, Wang Q. Plaque features and vascular geometry in basilar artery atherosclerosis. Medicine 2020;99:e19742.

13. Kim BJ, Kim HY, Jho W, Kim YS, Koh SH, Heo SH, Chang DI, Lee YJ. Asymptomatic Basilar Artery Plaque Distribution and Vascular Geometry. J Atheroscler Thromb 2019;26:1007-14.

14. Yu J, Zhang S, Li ML, Ma Y, Dong YR, Lou M, Feng F, Gao S, Wu SW, Xu WH. Relationship between the geometry patterns of vertebrobasilar artery and atherosclerosis. BMC Neurol 2018;18:83.

15. Wei H, Zhang M, Li Y, Zhao X, Canton G, Sun J, Xu D, Zhou Z, Chen S, Ferguson MS, Hatsukami TS, Li R, Yuan C. Evaluation of 3D multi-contrast carotid vessel wall MRI: a comparative study. Quant Imaging Med Surg 2020;10:269-82

16. Kerwin W, Xu D, Liu F, Saam T, Underhill H, Takaya N, Chu B, Hatsukami T, Yuan C. Magnetic resonance imaging of carotid atherosclerosis: plaque analysis. Top Magn Reson Imaging 2007;18:371-8.

17. Xu WH, Li ML, Gao S, Ni J, Zhou LX, Yao M, Peng B, Feng F, Jin ZY, Cui LY. In vivo high-resolution MR imaging of symptomatic and asymptomatic middle cerebral artery atherosclerotic stenosis. Atherosclerosis 2010;212:507-11.

18. Zhang DP, Peng YF, Zhang HL, Ma JG, Zhao M, Yin S, Wei TT. Basilar Artery Tortuosity Is Associated With White Matter Hyperintensities by TIMP-1. Front Neurosci 2019;13:836.

19. Kim BJ, Lee KM, Kim HY, Kim YS, Koh SH, Heo SH, Chang DI. Basilar Artery Plaque and Pontine Infarction Location and Vascular Geometry. J Stroke 2018;20:92-8.

20. Davies PF. Flow-mediated endothelial mechanotransduction. Physiol Rev 1995;75:519-60.

21. Dhawan SS, Avati Nanjundappa RP, Branch JR, Taylor WR, Quyyumi AA, Jo H, McDaniel MC, Suo J, Giddens D, Samady H. Shear stress and plaque development. Expert Rev Cardiovasc Ther 2010;8:545-56.

22. Reneman RS, Arts T, Hoeks AP. Wall shear stress--an important determinant of endothelial cell function and structure--in the arterial system in vivo. Discrepancies with theory. J Vasc Res 2006;43:251-69.

23. Malek AM, Alper SL, Izumo S. Hemodynamic shear stress and its role in atherosclerosis. JAMA 1999;282:2035-42.

24. Chen $\mathrm{W}, \mathrm{Xu} \mathrm{J}$, Chiu B. Fast segmentation of the femoral arteries from 3D MR images: A tool for rapid assessment of peripheral arterial disease. Med Phys 2015;42:2431-48.
25. Zhao X, Underhill HR, Zhao Q, Cai J, Li F, Oikawa M, Dong L, Ota H, Hatsukami TS, Chu B, Yuan C. Discriminating carotid atherosclerotic lesion severity by luminal stenosis and plaque burden: a comparison utilizing high-resolution magnetic resonance imaging at 3.0 Tesla. Stroke 2011;42:347-53.

26. Costopoulos C, Timmins LH, Huang Y, Hung OY, Molony DS, Brown AJ, Davis EL, Teng Z, Gillard JH, Samady H, Bennett MR. Impact of combined plaque structural stress and wall shear stress on coronary plaque progression, regression, and changes in composition. Eur Heart J 2019;40:1411-22.

27. Cheng C, Tempel D, van Haperen R, van der Baan A, Grosveld F, Daemen MJ, Krams R, de Crom R. Atherosclerotic lesion size and vulnerability are determined by patterns of fluid shear stress. Circulation 2006;113:2744-53.

28. Eshtehardi P, McDaniel MC, Suo J, Dhawan SS, Timmins LH, Binongo JN, Golub LJ, Corban MT, Finn AV, Oshinski JN, Quyyumi AA, Giddens DP, Samady H. Association of coronary wall shear stress with atherosclerotic plaque burden, composition, and distribution in patients with coronary artery disease. J Am Heart Assoc 2012;1:e002543.

29. Fan R, Tang D, Yang C, Zheng J, Bach R, Wang L, Muccigrosso D, Billiar K, Zhu J, Ma G, Maehara A, Mintz GS. Human coronary plaque wall thickness correlated positively with flow shear stress and negatively with plaque wall stress: an IVUS-based fluid-structure interaction multi-patient study. Biomed Eng Online 2014;13:32.

30. Wahle A, Lopez JJ, Olszewski ME, Vigmostad SC, Chandran KB, Rossen JD, Sonka M. Plaque development, vessel curvature, and wall shear stress in coronary arteries assessed by X-ray angiography and intravascular ultrasound. Med Image Anal 2006;10:615-31.

31. Kim JS, Nah HW, Park SM, Kim SK, Cho KH, Lee J, Lee YS, Kim J, Ha SW, Kim EG, Kim DE, Kang DW, Kwon $\mathrm{SU}, \mathrm{Yu} \mathrm{KH}$, Lee BC. Risk factors and stroke mechanisms in atherosclerotic stroke: intracranial compared with extracranial and anterior compared with posterior circulation disease. Stroke 2012;43:3313-8.

32. Sasamura H, Itoh H. Hypertension and arteriosclerosis. Nihon Rinsho 2011;69:125-30.

33. Gao X, Song J, Watase H, Hippe DS, Zhao X, Canton G, Tian F, Du R, Ji S, Yuan C, CARE-II Investigators. Differences in Carotid Plaques Between Symptomatic Patients With and Without Diabetes Mellitus. Arterioscler 
Thromb Vasc Biol 2019;39:1234-39.

34. Wei LM, Zhu YQ, Bao YQ, Lu HT, Zhang PL, Zhao YW, Li M, Zhao JG. Atherosclerosis in intracranial

Cite this article as: Deng S, Zheng J, Wu Y, Yang D, Chen H, Sun B, Xue Y, Zhao X. Geometrical characteristics associated with atherosclerotic disease in the basilar artery: a magnetic resonance vessel wall imaging study. Quant Imaging Med Surg 2021;11(6):2711-2720. doi: 10.21037/qims-20-1291 or extracranial vessels in diabetic patients and the association with stroke subtype. Quant Imaging Med Surg 2019;9:960-7. 


\section{Supplementary}

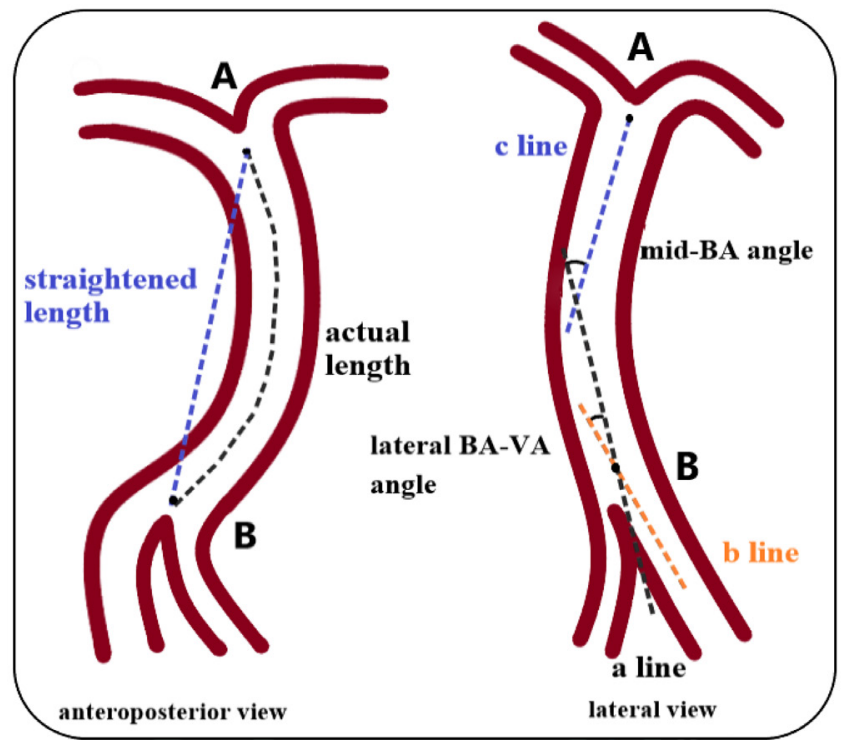

Figure S1 Illustration of the measurement of basilar artery geometry. A: top of BA; B: junction of both VAs. The picture shows a left convex BA. Tortuosity $=($ actual length/straightened length -1$) \times 100$. A straight line between A to B was used to determine BA convexity in the anteroposterior view. In the lateral view, the angle between the BA (a line) and the dominant VA (b line) was regarded as lateral BA-VA angle, and the angle between a line and c line was considered as lateral mid-BA angle. BA, basilar artery; VA, vertebral artery.

Table S1 Inter-observer and intra-observer reproducibility in measuring BA geometry

\begin{tabular}{lcc}
\hline & & ICC (95\% CI) \\
\cline { 2 - 3 } & \multicolumn{1}{c}{ Inter-observer } & Intra-observer \\
\hline Actual length & $0.916(0.827,0.960)$ & $0.926(0.847,0.965)$ \\
Straightened length & $0.920(0.834,0.962)$ & $0.919(0.834,0.962)$ \\
Tortuosity & $0.875(0.749,0.940)$ & $0.865(0.730,0.935)$ \\
Lateral BA-VA & $0.965(0.864,0.991)$ & $0.935(0.868,0.968)$ \\
Lateral mid-BA & $0.878(0.586,0.968)$ & $0.881(0.765,0.941)$ \\
\hline
\end{tabular}

BA, basilar artery; VA, vertebral artery; ICC, intraclass correlation coefficient; Cl, confidence interval. 